\title{
RETOS Y PROPUESTAS DE LA PROFESIÓN CONTABLE PARA EL DESARROLLO DE ECONOMÍAS SUSTENTABLES EN LATINOAMÉRICA
}

\author{
CHALLENGES AND PROPOSALS OF THE ACCOUNTING PROFESSION FOR THE \\ DEVELOPMENT OF SUSTAINABLE ECONOMIES IN LATIN AMERICA
}

José MARÍA GonZÁLEZ

Universidad Nacional de Entre Ríos Buenos Aires, Argentina ORCID: http://orcid.org/0000-0001-9945-9740 Correo electrónico: josemaria.gonzalezrube@gmail.com

Oscar Alfredo Díaz Becerra Pontificia Universidad Católica del Perú Lima, Perú ORCID: http://orcid.org/0000-0003-3313-0496 Correo electrónico: odiaz@pucp.edu.pe

Elsa Esther Choy Zevallos Universidad Nacional Mayor de San Marcos Lima, Perú

ORCID: http://orcid.org/0000-0001-8580-8495

Correo electrónico: echoyz@unmsm.edu.pe

[Recibido: 07/05/2019 Aceptado: 02/08/2019 Publicado: 28/08/2019]

\section{RESUMEN}

Los reportes con información no financiera, especialmente de carácter ambiental y social, mediante la utilización de diversos estándares internacionales han generado en la profesión contable latinoamericana importantes desafíos, tanto respecto de su conocimiento como también en la implementación en las diversas organizaciones, especialmente en las pequeñas y medianas empresas, que constituyen factores necesarios para el desarrollo económico sustentable de la región. El proyecto de investigación se desarrolló mediante una encuesta estructurada para identificar las actividades empresariales que impactan en el medio ambiente latinoamericano, lo cual afecta el desarrollo económico sustentable, también se buscó relevar la responsabilidad social de las organizaciones, a través del análisis de sus Balances Sociales o Memorias de Sostenibilidad. Como marco de referencia teórico, para este tipo de reportes, se analizan conceptos de Contabilidad, su evolución y especialmente de la denominada Contabilidad Económica y de nuevas propuestas como el enfoque tridimensional de la Contabilidad. Uno de los estándares más utilizados en nuestra región latinoamericana son las Guías de Iniciativa de Reporte Global (GRI), pero con escaso uso por parte de las pequeñas y medianas empresas, por lo que será un interesante desafío intentar realizar una propuesta para que se considere en la preparación de las características de nuestras Pymes latinoamericanas y, de esta forma, lograr que los contadores públicos tengan la correspondiente formación académica.

Palabras clave: Desarrollo; sostenible; contabilidad; economía; reportes.

\begin{abstract}
The reports with non-financial information, especially environmental and social, through the use of various international standards have generated significant challenges in the Latin American accounting profession, both in terms of their knowledge and in the implementation in the various organizations, especially in the small and medium-sized companies, which are necessary factors for the sustainable economic development of the Region. The research project was developed through a structured survey to identify business activities that impact the Latin American environment, affecting sustainable economic development and also to highlight the social responsibility of organizations, by analyzing their Social Balances or Sustainability Reports. As a framework of theoretical reference, for this type of reports Accounting evolution and concepts are analyzed, especially the so-called Economic Accounting as well as new proposals such as the three-dimensional approach to Accounting. One of the most widely used standards in our Latin American region are the GRI Guidelines, but with little use by Small and Medium Enterprises. Therefore, it will be an interesting challenge to try to make a proposal that takes into account the characteristics of our Latin American SMEs in its preparation and in this way ensure that the public accountants have the corresponding academic training.
\end{abstract}

Keywords: Development; sustainable; accounting; economics; reports.

( - Los autores. Este artículo es publicado por la Revista Quipukamayoc, Universidad Nacional Mayor de San Marcos. Este es un artículo de acceso abierto, distribuido bajo los términos de la Licencia Creative Commons Atribución-NoComercial-Compartirlgual 4.0 Internacional.(http://creativecommons.org/licenses/by-nc-sa/4.0/), que permite el uso no comercial, distribución y reproducción en cualquier medio, siempre que la obra original sea debidamente citadas. 


\section{INTRODUCCIÓN}

La mayoría de empresas en Latinoamérica pone mayor énfasis en la preparación y presentación de informes financieros para uso de la gerencia. Son pocas las empresas que recurren a la elaboración de algún tipo de reporte no financiero, que incorpore información de tipo ambiental y social, y que estos sean preparados sobre la base de estándares internacionales. En este escenario, la profesión contable latinoamericana se enfrenta a un importante desafío: la necesidad de establecer mecanismos para que las organizaciones, especialmente, las pequeñas y medianas empresas, incorporen algún tipo de reporte no financiero y que permita conocer el impacto y las acciones que llevan a cabo, en relación al desarrollo económico sostenible de la región.

En este contexto, se presenta este trabajo de investigación, entre cuyos objetivos podemos destacar, cuál será el rol de la profesión contable latinoamericana, en relación con los diversos requerimientos a las organizaciones en materia de presentación de Informes Financieros, Ambientales, Económicos y más recientemente los Reportes Integrados, especialmente en relación con la aplicación generalizada en las pequeñas y medianas empresas (pymes).

También constituyen objetivos de este trabajo, generar un espacio de reflexión sobre el futuro de la profesión del contador público, en relación con los reportes de los sistemas de información, con un pensamiento innovador, en cumplimiento de su responsabilidad social, para modificar algunas visiones sobre la profesión contable en su conjunto, Gray y Bebbington (2006) expresaron lo siguiente:

La investigación sugiere que los contadores no son vistos como una fuente principal de innovación, en la organización, generalmente, y en la respuesta organizacional al ambiente en particular. Además, si bien los contadores se ven a sí mismos como potencialmente innovadores, es una innovación forzada al nivel de información financiera existente [...] Sin embargo, las rápidamente cambiantes exigencias sobre la organización generarán una demanda urgente por cambios en las actividades de los contadores. Solamente los que tienen visión de largo plazo ya están respondiendo a esto. (p. 15)

Para desarrollar esta investigación de este trabajo, se implementó una encuesta estructurada para identificar las actividades empresariales que impactan en el medio ambiente latinoamericano y la responsabilidad social de las organizaciones, como también identificar las regulaciones en los países latinoamericanos en materia de Reportes de Sostenibilidad. Pero, ante la escasa respuesta de la encuesta, el trabajo ha sido desarrollado en función de la información correspondiente a los países a los cuales representan sus autores, con un enfoque que comprenda las realidades profesionales de los países latinoamericanos miembros de Asociación Interamericana de Contabilidad.

\section{Contabilidad ambiental}

Ubicación en el campo del conocimiento, objeto y naturaleza

La ubicación en el campo del conocimiento de la contabilidad o disciplina contable ha generado diversas posiciones doctrinarias y ha evolucionado en su concepción, especialmente por la relevancia que ha ido adquiriendo la utilización de la información contable en las organizaciones en general, para los diversos usuarios o grupos de interés.

Para García, citado por Pahlen y Fronti (2004) refieren que:

La Contabilidad es una ciencia factual, cultural, aplicada, que se ocupa de explicar y normar las tareas de descripción, principalmente cuantitativa, de la existencia y circulación de objetos, hechos y personas diversas de cada ente u organismo social y de la proyección de los mismos en vista al cumplimiento de metas organizacionales a través de sistemas basados en un conjunto de supuestos básicos y adecuados a cada situación. (p. 164)

El citado autor ha diferenciado los siguientes segmentos de la contabilidad: contabilidad patrimonial o financiera, contabilidad gerencial, contabilidad gubernamental, contabilidad económica; $y$, contabilidad social (macro y micro). Sobre la base de estos segmentos ha considerado los siguientes modelos contables: patrimoniales o financieros, gerenciales, gubernamentales, macroeconómicos, microsociales y macrosociales.

Para Biondi (2007) el uso de la Contabilidad, es muy diverso y constituye en realidad una base de datos que se puede utilizar con distintos objetivos. Para este autor, ese conjunto de información, puede denominarse Partes o Segmentos de la Contabilidad, distinguiendo los siguientes destinatarios:

i. Terceros: vinculados con el ente con el cual operan. El segmento contable que procura la información útil para que los terceros estén enterados de cómo marcha la empresa y si existe algún riesgo por operar con ella se denomina Contabilidad Financiera o Patrimonial y está regulado por normas de aplicación que explican cómo y por qué se realizan los estados contables de manera uniforme para todas las empresas.

ii. El propio Ente que requiere información pormenorizada para tomar decisiones. Este segmento contable no está regulado por normas, normalmente se la denomina Contabilidad de Gestión o Gerencial. 
iii. Los organismos nacionales para recopilar información macroeconómica. Generalmente este segmento se conoce como Contabilidad Económica y pretende recopilar información que interesa a todos los habitantes de un país.

iv. Control de los ingresos y egresos del Estado. Este segmento se lo denomina Contabilidad de las Finanzas Públicas o Contabilidad Pública y tiene mucha importancia para la rendición de cuentas del manejo de fondos por parte del Estado.

v. Ingresos y egresos específicos derivados del mantenimiento del equilibrio ecológico. Se denomina Contabilidad Ecológica.

vi. Responsabilidad derivada de la vida en sociedad. El Estado y las empresas invierten fondos para mejorar la calidad de vida de la población. El segmento se denomina Contabilidad Social.

Para Mejía, Montilla, Montes, y Mora (2016) la Contabilidad Ambiental, se define como:

La ciencia social aplicada, que estudia la valoración cualitativa y cuantitativa de la existencia $y$ circulación de la riqueza ambiental controlada por las organizaciones, utilizando diversos métodos que le permite evaluar la gestión que la organización ejerce sobre la riqueza ambiental, con el fin de contribuir a la acumulación, generación, distribución y sostenibilidad integral de la misma, siendo por ende la Contabilidad, para estos autores, una ciencia aplicada. (p. 60)

Con un enfoque en la Teoría Tridimensional de la Contabilidad, los autores Mejía y Serna (2016) concluyen que:

Las organizaciones en sus dinámicas económicas generan impactos ambientales y sociales que no presentan en sus estados contable, situación que lleva a formular la necesidad de adicionar a la información económica tradicional, informes contables ambientales y sociales que permitan una rendición de cuentas pública de la organización, que revele a la sociedad los impactos totales que genera con su actuación. La representación contable de la riqueza natural no garantiza su protección y cuidado, pero sin su contabilización no habrá preservación. (p. 101)

Formación de los Profesionales en Ciencias Económicas.

Las Normas Internacionales de Formación en Contaduría, acreditan esta posición atenta a que: conforme a las Normas Internacionales de Formación en Contaduría 1-8 (International Education Standards / IES 1-8) emitidas por la Federación Internacional de Contadores, (IFAC) prevé en los programas de formación y desarrollo las capacidades y competencias necesarias para su actuación en el proceso de instrumentación y aseguramiento del Balance Social.

Así, entre los requerimientos de la IES 2 se incluye en relación con esta temática los siguientes aspectos relevantes: interpretar los informes que incluyen datos no financieros, por ejemplo, los informes de sostenibilidad e informes integrados; comprensión de conceptos de gobierno corporativo y ética empresarial; comprensión de las cuestiones ambientales y el desarrollo sostenible; y emisión de informes.

Actividades empresariales que impactan en el medio ambiente latinoamericano. Responsabilidad Social.

De forma general, en nuestra economía latinoamericana, el crecimiento vegetativo de la población, la volatilidad de los precios del valor de los recursos naturales y su utilización descontrolada, pueden considerarse factores que impiden el crecimiento económico sustentable de nuestros países.

Desde el punto de vista económico, se ha considerado la degradación ambiental como un caso particular de las fallas de mercado, las que se refieren a la divergencia entre los precios de mercado y los precios que tendrían que tener los bienes ambientales para alcanzar un óptimo; por lo que las variaciones en cantidad y calidad de las funciones ambientales, tanto en el corto como en el largo plazo, generan degradación e interrelaciones ambientales, sociales y económicas, que derivarán en la contracción de la frontera de posibilidad de producción.

\section{Experiencias en el caso de Argentina.}

En el caso de Argentina, existen actividades de alto impacto en el medioambiente, como son la minería y la agricultura. También hay otras actividades que fueron relevadas con información mediante encuestas, informando las siguientes jurisdicciones:

Provincia de Tierra del Fuego. El organismo Informante fue el Consejo Profesional de Ciencias Económicas - Cámara Rio Grande. En la Tabla 1 se detalla las principales actividades económicas de la provincia, con los impactos más frecuentes de su actividad (ver tabla 1).

Provincia de Córdoba. Se ha identificado como actividades que impactan en el medio ambiente, la agropecuaria (soja), agro-negocios transgénicos (semillas, fertilizantes y agro-tóxicos), y extracción de maderas (desforestación, tala indiscriminada de árboles).

En el caso de la provincia, los resultados son variados. Las dos primeras se extienden en el sur, sur-este, este, nor-este y centro de la provincia. La tercera, afecta especialmente al 
Tabla 1

Impacto ambiental en la provincia de Tierra del Fuego.

\begin{tabular}{|c|c|c|c|c|c|c|c|}
\hline \multirow[b]{2}{*}{ Actividad } & \multirow[b]{2}{*}{ Departamento } & \multicolumn{5}{|c|}{ Tipo de Impacto Ambiental } & \multirow{2}{*}{$\begin{array}{c}\text { Notas } \\
\text { periodísticas } \\
\text { sobre impactos } \\
\text { ambientales }\end{array}$} \\
\hline & & $\begin{array}{l}\text { Consumo } \\
\text { de recursos } \\
\text { naturales }\end{array}$ & $\begin{array}{c}\text { Alteración } \\
\text { de uso de } \\
\text { los espacios }\end{array}$ & $\begin{array}{l}\text { Generación de } \\
\text { residuos sólidos } \\
\text { contaminantes }\end{array}$ & $\begin{array}{l}\text { Generación de } \\
\text { residuos líquidos } \\
\text { contaminantes }\end{array}$ & $\begin{array}{l}\text { Generación de } \\
\text { residuos gaseosos } \\
\text { contaminantes } \\
\text { (distintos de CO2) }\end{array}$ & \\
\hline \multicolumn{8}{|l|}{$\begin{array}{r}\text { 1.- Actividad } \\
\text { extractiva }\end{array}$} \\
\hline $\begin{array}{r}\text { Petróleo y } \\
\text { Gas }\end{array}$ & Río Grande & Si & & & Si & & Si \\
\hline $\begin{array}{r}\text { Pesca } \\
\text { marítima }\end{array}$ & Ushuaia & Si & & & & & Si \\
\hline Acuicultura & Almanza & & Si & & & & \\
\hline $\begin{array}{r}\text { Minería } \\
\text { de tercera } \\
\text { categoría }\end{array}$ & $\begin{array}{c}\text { Río Grande - } \\
\text { Tolhuin }\end{array}$ & Si & & & & & Si \\
\hline Forestal & Tolhuin & $\mathrm{Si}$ & & & & & \\
\hline \multicolumn{8}{|l|}{$\begin{array}{r}\text { 2.- Actividad } \\
\text { industrial }\end{array}$} \\
\hline $\begin{array}{r}\text { Industrial } \\
\text { química } \\
\text { (agro } \\
\text { tóxicos) }\end{array}$ & Río Grande & & & & Si & & Si \\
\hline $\begin{array}{r}\text { Industria } \\
\text { textil }\end{array}$ & Río Grande & & & & Si & & Si \\
\hline $\begin{array}{l}\text { Industrial } \\
\text { electrónica }\end{array}$ & $\begin{array}{c}\text { Río Grande y } \\
\text { Ushuaia }\end{array}$ & & & Si & & & Si \\
\hline $\begin{array}{l}\text { Industrial } \\
\text { plástica }\end{array}$ & Río Grande & & & & & Si & Si \\
\hline
\end{tabular}

Fuente: Encuesta CPCE Tierra del Fuego - Cámara Río Grande.

departamento Calamuchita. No hay antecedentes de presentación de Balances Sociales o Reportes de Sostenibilidad y no hay normativa que requiera la presentación los mismos.

\section{Actividad minera}

Se presenta el caso de la empresa Minera Alumbrera, cuya actividad principal es la extracción de minerales metalíferos no ferrosos, excepto minerales de uranio y torio. La explotación se ubica en un yacimiento en la provincia de Catamarca, mientras que la planta de filtros se realiza en Cruz del Norte, provincia de Tucumán y el envío en el Puerto Alumbrera, en el Puerto General San Martín, provincia de Santa Fe. La empresa presenta el Informe de Sostenibilidad 2015, elaborado según la Guía G4 de Global Reporting Initiative; el Pacto Mundial de Naciones Unidas; Estándar AA1000 SES de Accountability; así como los lineamientos del Consejo Empresario Argentino para el Desarrollo Sostenible (ver tabla 2).

La Minera Alumbrera menciona en su página web que:

En Alumbrera buscan mitigar las posibles consecuencias mediante programas ambientales, tales como la revegetación con especies nativas, la reubicación de cardones recuperados de zonas operativas o la reforestación, además de controles para evitar la afectación del agua, suelo y aire, más allá de los límites de concesión. Protegen la biodiversidad también a través de monitoreo de la flora y la fauna del área del yacimiento. Durante 2015 se produjeron plantines de algarrobos, arribando a un total de 5900 plantines en canteros de producción y 500 en el área de rustificación, listos para el trasplante al terreno. El consumo de agua, energía y gasoil en 2015 se realizó de acuerdo con lo planificado, lo que se refleja en el cumplimiento del $100 \%$ de los indicadores de ecoeficiencia. (Minera Alumbrera, 2017)

Actividad Agropecuaria

Se presenta el caso de la empresa Los Grobo Agropecuaria S.A. (LGA) ubicada en la Ruta 5 km 309 de la Provincia de Buenos Aires, donde Los Grobo Agropecuaria (2017) refieren que:

La empresa ofrece una amplia plataforma de negocios que comprende la producción agropecuaria, la 
Tabla 2

Información relativa al Impacto en el medio ambiente.

\begin{tabular}{|c|c|c|c|}
\hline MEDIO AMBIENTE & 2013 & 2014 & 2015 \\
\hline Consumo directo de energía & 865822900 KWh & $847376421 \mathrm{KWh}$ & $852427903 \mathrm{KWh}$ \\
\hline Consumo total de energía & 898017854 KWh & $852235000 \mathrm{KWh}$ & $855631393 \mathrm{KWh}$ \\
\hline Consumo total de agua & $24506878 \mathrm{~m} 3$ & $23451000 \mathrm{~m} 3$ & $22588089 \mathrm{~m} 3$ \\
\hline Total de agua reciclada y reutilizada & $64562000 \mathrm{~m} 3$ & $62324000 \mathrm{~m} 3$ & $59040298 \mathrm{~m} 3$ \\
\hline $\begin{array}{l}\text { Emisiones directas de efecto invernadero (Eq. } \\
\text { de CO2 por millón de toneladas movidas) }\end{array}$ & 1,78 & 1,71 & 1,92 \\
\hline Emisiones de dióxido de sulfuro por chimeneas & & & No reportan \\
\hline Tierras intervenidas & 63,52 hectáreas & 65,82 hectáreas & 65,59 hectáreas \\
\hline Tierras rehabilitadas & 0 hectáreas & 2,72 hectáreas & 6,42 hectáreas \\
\hline
\end{tabular}

Fuente: Informe Sostenibilidad 2015 Minera Alumbrera.

comercialización de granos y oleaginosas, así como la prestación de servicios, incluyendo la provisión de agro insumos, la asistencia técnica y financiera a pequeños productores. La empresa produce soja, maíz y trigo, en tierra arrendada o en sociedad, tercerizando la mano de obra y la maquinaria. Proveen una plataforma segmentada de servicios que incluye -entre otros- la provisión de insumos, la comercialización de granos, el financiamiento de pequeños productores, el almacenamiento y la realización de operaciones logísticas para la venta de granos. (Los Grobo Agropecuaria, 2017, p.1)

La empresa presenta el Reporte de Sustentabilidad 20142015, el cual se basa en indicadores referidos a temas ambientales, sociales, económicos y financieros relacionados con la Empresa en cuestión. Utilizaron los lineamientos de la Guía G4 del GRI para la elaboración de Reportes de Sustentabilidad.

Se busca producir en forma flexible para poder adaptarse a los cambios y las exigencias, tanto locales como internacionales, pero a la vez busca mejorar en forma continua su relación con el medio ambiente a través de la adopción de mejores tecnologías, procesos dinámicos, formas de trabajo conjunto y consensuado. Específicamente en referencia a este acuerdo mundial, desde LGA se implementan las siguientes iniciativas:

- No se realizan desmontes, ni remoción de vegetación nativa.

- La agricultura se realiza en siembra directa, propiciando un equilibrio en el suelo.

- Se realiza un monitoreo semestral de la materia orgánica en el suelo, en caso de balances negativos se toman medidas para revertirlo.
- Se realiza agricultura por ambientes haciendo un uso más eficiente de los fertilizantes.

- Se cuenta con un manejo integrado de plagas, con métodos complementarios los cuales se aplican en tres etapas: prevención, observación y aplicación. Es un método ecológico que aspira a reducir o eliminar el uso de plaguicidas.

- No se hace agricultura en campos con remoción de hábitat nativo después del año 2008.

- Se verifica si los establecimientos se encuentran o interfieren con áreas de conservación a fin de determinar inconsistencias desde el punto de vista de la pérdida de la biodiversidad y de especies y evaluar las posibles afectaciones por la realización de los cultivos mencionados sobre áreas sensibles.

- En los centros de acopio y almacenaje se reemplazan sistema de elevadores que consumen menos energía.

- Para el transporte de materias primas y productos, se solicita la Verificación Técnica Vehicular a los transportes de carga contratados y a los propios. Además, se propicia la contratación de vehículos que emiten menor cantidad de GEI.

- Se realiza una planificación y control de la logística de los recorridos para hacer un uso más eficiente de los recursos.

- La empresa cuenta con sectores cuyas actividades implican un alto grado de movilidad, a partir de esto se proponen ahorros en el consumo de manera indirecta, a través de la implantación de sistemas de comunicación como conferencias y video conferencias virtuales. 


\section{Experiencias en el caso de Perú.}

\section{Actividad minera}

Se presenta el caso de la Compañía Minera Buenaventura, la cual se dedica a actividades extractivas de minerales, como el oro y plata. Se encuentra ubicada en la provincia de Castilla, en Arequipa. La empresa presenta el Reporte de Sostenibilidad Corporativa, el cual forma parte del Manual para la Preparación de Memorias Anuales emitido por la Superintendencia del Mercado de Valores, vigente desde el año 2016. La norma es de aplicación obligatoria para las empresas que cotizan en el mercado de valores.

En la publicación Sophimania (2015), se señala que las cuatro actividades más contaminantes en el país son:

- Conversión de bosques y pasturas, o cambio de uso de suelo, producto de la deforestación de la Amazonía para ampliar los suelos agrícolas, que a nivel nacional es principal fuente de emisión con un 40,9\%; se pierden miles de hectáreas por la tala ilegal, minería, entre otros.

- Sector Energía, con 21,2\% de los GEI, principalmente por las emisiones del transporte terrestre.

- Agricultura, con $18,9 \%$, por emisión de metano por fermentación entérica y suelos agrícolas por emisión N02 (GEI).

- Procesos industriales, con 6,6\%, principalmente por la producción de metal hierro y acero, que contribuye con emisiones.

Según López (2017), informa que las opiniones de cinco especialistas en temas ambientales coinciden en señalar que las actividades de alto impacto ambiental son:

a. Derrames petroleros en la Amazonía, donde la empresa responsable es Petroperú. En el 2016 los derrames en la Amazonía peruana fueron: en Imaza, Amazonas, 2000 barriles y 1900 afectados; en Morona, Loreto, 1000 barriles y 2543 afectados; en Barranca, Loreto, 600 barriles y 900 afectados. En el 2015, derrame en Trompeteros(Loreto), 200 barriles, 450 afectados, empresa encargada Pluspetrol. En el 2014, derrame en Urarinas, Loreto, 3000 barriles y 60,000 afectados; considerándose el desastre de mayor impacto.

b. Incendios forestales zona Norte, en los cuales, en el año 2016, el Servicio Nacional de Áreas Naturales Protegidas por el Estado (Sernanp) informó que los incendios forestales afectaron zonas de diez regiones del Perú, entre las cuales se encuentran zonas norte- ñas protegidas en Cajamarca, Lambayeque y Tumbes. Los incendios a consecuencia de altas temperaturas, fuertes vientos y clima seco.

c. Sequía zona Sierra y Norte, principalmente en la Siera de Ayacucho, la sequía afecta la ganadería y agricultura con muerte de animales y no crecimiento de los cultivos; algunos pueblos afectados son los centros poblados del valle de Sondondo.

Necesidad de información de los diversos grupos de interés. Caso peruano

Las organizaciones ponen gran énfasis en la preparación y presentación de su información financiera sobre la base de estándares internacionales de contabilidad. En particular, aquellas que se encuentran listadas en bolsa. Por su parte, las demás organizaciones, suelen poner menor énfasis en el cumplimiento de la información financiera para los usuarios externos y se enfocan más en la información requerida por la gerencia para la toma de decisiones.

Es en este contexto que los informes no financieros, como el caso de los reportes de sostenibilidad, no han tenido mucha atención por parte de las empresas peruanas y recién, con la promulgación de la Resolución SMV Nº332015-SMV/01 del 15 de diciembre de 2015, se establece la obligatoriedad de incorporar un anexo adicional a la sección IV de la Memoria, denominada Reporte de Sostenibilidad Corporativa.

Según lo establecido en esta nueva disposición, la sección A del reporte debe informar sobre la implementación de acciones de Sostenibilidad Corporativa. Incluye seis preguntas, entre las que se debe informar, por ejemplo, si la empresa se ha insertado de manera voluntaria a estándares de buenas prácticas respecto a la Sostenibilidad Corporativa, si tiene políticas vinculadas a los colaboradores, entre otros. En la sección B del reporte de debe detallar los grupos de interés de la sociedad, así como las acciones implementadas durante el ejercicio y que se encuentran vinculadas al impacto de sus operaciones en el desarrollo social y el medio ambiente.

La información estadística del reporte de sostenibilidad corporativa de la SMV $(2017$, p.5) indica que por el año 2016, un total de 208 empresas presentaron dicho reporte, de las cuales, 86 han declarado que se han adherido voluntariamente a estándares de buenas prácticas en materia de Sostenibilidad Corporativa, mientras que 122 no lo han hecho en forma voluntaria. Otra información relevante está referida a si la empresa tiene una política corporativa relacionada al impacto de sus actividades en el medio ambiente, 116 empresas respondieron que sí, siendo el grupo más representativo el de las empresas del sector industrial. 
Antes de la vigencia del Reporte de Sostenibilidad Corporativa, encontramos empresas que presentaban algún tipo de reporte de sostenibilidad, como es el caso de Sodimac Perú S.A., Alicorp S.A.A., Yanacocha S.R.L., PwC Perú, Cosapi S.A., Compañía Minera Antamina S.A., Sociedad Minera el Brocal, entre otras. Esto evidencia el interés de algunas organizaciones de proporcionar información no financiera, sin que una norma los obligue a ello.

El estudio, Corrientes de Cambio, elaborado por KPMG, contiene información del año 2015 sobre los reportes de sostenibilidad en 45 países del mundo, incluido Perú. En este estudio se indica que Perú se encuentra por debajo del promedio de los reportes que se generan a nivel mundial, lo cual se explica por el hecho de que, a la fecha de elaboración del estudio, en Perú aún no era obligatorio la inclusión de este tipo de información en los reportes anuales. (KPMG, 2016, p.7).

Instrumentos utilizados para la generación de reportes de sostenibilidad en los países latinoamericanos.

El Trabajo Interamericano Balance Social y Gobierno corporativo de las empresas latinoamericanas presentado en la CIC realizada en el año 2015 en Punta Cana, se incluyeron entre los diversos instrumentos en los países latinoamericanos a los siguientes:

Organización Internacional para la Estandarización (normas ISO)

La ISO 26000 aborda asuntos de responsabilidad social como: gobierno de la organización, derechos humanos, prácticas laborales; medio ambiente, prácticas justas de operación; asuntos de consumidores; y participación activa y desarrollo de la comunidad.

La ISO 14001 establece, documenta, implanta, mantiene y mejora continuamente una política ambiental y un sistema de gestión ambiental y determina cómo se cumplirá con dichos requisitos.

\section{Institute for Social and Ethical Accountability- ISEA -Gran} Bretaña.

En noviembre de 1999, esta organización emitió las Series AA 1000 (Accountability1000) con el objeto de integrar la gestión, auditoría y la comunicación en los aspectos relativos a la responsabilidad social de las empresas, instituciones de la sociedad civil y organismos públicos.

Global Reporting Initiative (GRI)

Esta organización surgió por iniciativa del Coalition for Environmentally Responsible Economies (CERES) en co- laboración con el Programa de las Naciones Unidas para el Medio Ambiente (PNUMA). El GRI, pretende confeccionar, divulgar o transmitir un conjunto de pautas para la preparación de Memorias de Sostenibilidad.

\section{Social Accountability International}

Esta organización, en el año 1997, emite la norma SA 8000 (Social Accountability 8000 - USA) la cual es una norma uniforme y auditable mediante un sistema de verificación externo. Puede ser empleada voluntariamente por las empresas, independientemente del tamaño, lugar de ubicación o sector en donde opera, para demostrar su compromiso con las mejores prácticas de responsabilidad social respecto del ámbito interno. Introduce un sistema de gerenciamiento social (SMS) e incluye los principales derechos de los trabajadores.

Instituto de Empresas e Responsabilidade Social (ETHOS)

El Instituto Ethos es una organización sin fines de lucro, líder en Brasil, y una referencia en responsabilidad social empresaria que presenta los Indicadores Ethos de Responsabilidad Social Empresarial, para ofrecer a las empresas una herramienta que las auxilie en el proceso de profundización de su compromiso con la responsabilidad social y el desarrollo sostenible.

\section{Desarrollo de la responsabilidad social (DERES)}

DERES es una organización integrada por empresas, organizaciones empresariales e instituciones que articula distintos esfuerzos en procura de una concientización de todos los actores de la sociedad sobre el rol de los empresarios y la necesidad de la incorporación por parte de estos de los conceptos de la Responsabilidad Social en la gestión de sus organizaciones.

\section{Comité de Integración Latino Europa-América (CILEA)}

Para este Comité, los modelos y guías para la preparación de Balance Social y Ambiental sufren cambios vertiginosos, producto del cada vez mayor interés en poseer información de las organizaciones que no se limite solamente a la faz financiera, sino también que evidencie su compromiso social y ambiental. Así, nos encontramos con el desafío de mostrar el comportamiento de las organizaciones respecto del capital humano, social, intelectual y natural. (Gonzales, 2015, p. 3)

\section{Reporte Integrado}

El International Integrated Reporting Council (IIRC) es una organización mundial sin fines de lucro constituida en Inglaterra y Gales. Además, es una coalición global de: 
reguladores, inversores, empresas, reguladoras de estándares, emisores de normas profesionales contables y organizaciones no gubernamentales que comparte la visión de que la comunicación de la creación del valor deberán ser el próximo paso en la evolución del reporting corporativo.

Instrumento más utilizado en general en los países latinoamericanos: GRI

En la Guía GRI versión 4, se incluye un glosario y se brindan dos opciones de conformidad, la esencial y la exhaustiva. Los contenidos básicos específicos se organizan en tres categorías: Económica, Ambiental y Social. Los datos de cada aspecto material pueden presentarse como indicadores o como información sobre el enfoque de gestión.

La organización Global Reporting Initiative (2016) menciona:

Los Estándares GRI están diseñados principalmente para ser usados en conjunto a la hora de elaborar informes de sostenibilidad centrados en temas materiales. Los tres Estándares Universales son aplicables a cualquier organización que prepare un informe de sostenibilidad. Además, las organizaciones seleccionan de entre los Estándares temáticos para informar acerca de sus temas materiales, ya sean temas económicos, ambientales o sociales. La elaboración de informes con los Estándares GRI aporta una perspectiva general y equilibrada de los temas materiales de una organización, de los impactos relacionados y de cómo los gestiona. (p.2)

Regulaciones en los países latinoamericanos en materia de Reportes de Sostenibilidad

Argentina. No se han emitido disposiciones legales con alcance nacional que requiera a las diversas entidades y organizaciones para que emitan Reportes de Sostenibilidad. En cuanto a la Constitución Nacional Argentina l, menciona:

Artículo 41- [...] El daño ambiental generará prioritariamente la obligación de recomponer, según lo establezca la ley. Las autoridades proveerán a la protección de este derecho, a la utilización racional de los recursos naturales, a la preservación del patrimonio natural y cultural y de la diversidad biológica, y a la información y educación ambientales. [...]. Se prohíbe el ingreso al territorio nacional de residuos actual o potencialmente peligrosos, $y$ de los radiactivos.

Perú. El 15 de diciembre de 2015 se promulgó la Resolución SMV N ${ }^{\circ}$ 033-2015-SMV/01, mediante la cual se establece la obligatoriedad de incorporar un anexo adicional a la sección IV de la Memoria anual. Este anexo es denominado "Reporte de Sostenibilidad Corporativa" y debe ser incluido por las empresas a partir de la información que se presente del año 2016. El ámbito de aplicación es para las empresas peruanas que se encuentran bajo la supervisión de la Superintendencia del Mercado de Valores.

La Constitución Política del Perú, en el numeral 22 de su artículo segundo, incluye el derecho fundamental e irrenunciable a gozar de un ambiente adecuado y equilibrado para el desarrollo de la vida. Adicionalmente a esta normativa, se cuenta con otras normas relativas a protección del medio ambiente.

Contribución de la profesión contable latinoamericana en el desarrollo de economías sustentables

En relación con la Junta de Normas Internacionales de Contabilidad (IASB, por sus siglas en inglés), resulta de interés considerar el proyecto reportes corporativos lo tratado en su reunión de marzo del año 2017. El propósito de dicho trabajo fue buscar opiniones de la Junta, con el fin de realizar una contribución más activa en el área de reporting corporativo en base al análisis previsto en AP 28A.

En el informe de retroalimentación se observó que, de las respuestas recibidas en relación con el balance y el alcance de las actividades de la Junta, algunos inversionistas ponen mayor énfasis que otros en asuntos que amplíen los reportes corporativos y las actividades de la Junta para incluir temas como capital humano e intelectual, el cambio climático y la presentación de informes para entidades que coticen en un mercado no regulado. Varios grupos ambientalistas también dijeron que la presentación de informes sobre el cambio climático era un tema de creciente importancia.

También, el Informe de retroalimentación señaló que algunas de las implicancias del cambio climático se enmarcan dentro de proyectos activos o de investigación con infraestructura de tuberías y/o ductos, como provisiones, actividades extractivas y mecanismos de precios de los contaminantes. La Junta no agregó a su plan de trabajo un proyecto más amplio que incluya todos los aspectos del cambio climático. La Junta no ha identificado en esta etapa ninguna otra implicancia financiera del cambio climático que deba ser reportada y que requiera la fijación de normas en los próximos cinco años.

El Staff ha limitado las opciones a aquellas que creen mejor encajan dentro de la visión de los administradores, quienes entienden que la Junta no debería ampliar el alcance de su trabajo a áreas fuera de los límites tradicionales de los reportes financieros, pero que deberían establecer una visión clara acerca de cómo sus estándares u otros pronunciamientos, encajen hacia reportes más amplios.

La escasa preparación por parte de las pymes de Balances Sociales o Reportes de Sostenibilidad siguen requiriendo 
la atención de la profesión contable latinoamericana para continuar bregando por su utilización, como también necesita realizar los esfuerzos necesarios para alcanzar un estándar de calidad que permita cumplir con los objetivos del desarrollo económico sostenible. También será necesario que considere la preparación por parte de las pymes de Reportes Integrados, y sus características principales, sin descuidar los objetivos de dicho tipo de reportes. Si tenemos en cuenta los antecedentes en materia de normas contables de carácter financiero, el ejemplo más relevante son las NIIF para pymes.

\section{CONCLUSIONES}

El desarrollo sostenible se basa en tres factores: sociedad, economía y medio ambiente; y puede interpretarse como el conjunto de actividades económicas destinadas a satisfacer las necesidades de las generaciones presentes, sin comprometer las posibilidades de las generaciones del futuro para atender sus propias necesidades. En las pymes no se ha logrado esta concientización y necesidad de preparar información que contemple el objetivo de desarrollo sostenible.

La Asociación Interamericana de Contabilidad (AIC) y todos sus organismos miembros, deberán continuar realizando el esfuerzo para instalar en la profesión contable latinoamericana la necesidad de que las Pymes confeccionen y publiquen sus Balances Sociales o Memorias/ Reportes de Sostenibilidad asimismo y que celebre un convenio de cooperación con GRI para el desarrollo de una Guía de implementación para las pymes latinoamericanas, que contemple las características y actividades propias de la región, de forma similar, como lo han realizado otras organizaciones.

Las universidades y entidades académicas deben promover que en los planes de estudios de las carreras de contador público, contemplen asignaturas relativas a la preparación y verificación de Balances Sociales y/o Memorias o Reportes de Sostenibilidad, y además, que la profesión contable latinoamericana considere la utilización de los reportes integrados en las pymes, con el desarrollo de criterios con un enfoque hacia las mismas.

\section{REFERENCIAS BIBLIOGRÁFICAS}

Biondi, M. (2007). La Contabilidad: un Sistema de Información. Buenos Aires: ERREPAR.

Global Reporting Initiative (2016) Pequeñas Empresas. Grandes Impactos. Organización Internacional de Empleadores (OIE). Recuperado de: https://www.globalreporting.org/standards/gri-standards-translations/ gri-standards-spanish-translations-download-center/
Gonzales, J. (2015). Balance Social y Gobierno corporativo en las empresas latinoamericanas. Trabajo presentado en la XXI Conferencia Interamericana de Contabilidad. Buenos Aires: Asociación Interamericana de Contabilidad.

Gray, R., \& Bebbington, J. (2006). Contabilidad y Auditoría Ambiental. Segunda Edición. Santa Fe de Bogotá: ECOE Ediciones.

KPMG (2016). Corrientes de Cambio. Reporte sobre la encuesta de Sostenibilidad KPMG 2015. Lima: KPMG. Recuperado de: https://assets.kpmg/content/dam/ kpmg/pe/pdf/RS-Corrientes-de-Cambio.pdf

López, M. (2017) Perú: problemas ambientales que deben resolverse en el 2017. Lima: Plataforma Mongabay Latam.

Los Grobo Agropecuaria (2017). Bell Investment. Buenos Aires: Los Grobo. Recuperado de: https://www.bellbursatil.com/research-informacion-detalle/190-los-grobo-agropecuaria.

Mejía, E., Montilla, O., Montes, C., \& Mora, G. (2016) Investigaciones y Experiencias en Economía Solidaria: México-Colombia. México D.F.: Universidad Autónoma Chapingo.

Mejía, E., \& Serna, C. (2016) Contabilidad Ambiental: enfoque de publicaciones en Colombia (2009-2012). Revista Electrónica de Investigación en Ciencias Económicas. Armenia: Universidad de Quindio.

Minera Alumbrera (2017). Minera Alumbrera: Cuidado del Medio Ambiente. Buenos Aires: Argentina Ambiental. Recuperado de: http://argentinambiental.com/notas/ noti-empresas/minera-alumbrera-cuidado-del-medio-ambiente/

Pahlen, R., \& Fronti, L. (2004) Contabilidad social y ambiental. Buenos Aires.: Ediciones Machi.

Sophimania (2015) Contaminación y Salud Ambiental. Recuperado de: https://sophimania.pe/

Superintendencia del Mercado de Valores (2017). Información estadística del Reporte de Sostenibilidad Corporativa 2016. Lima: SMV. Recuperado de: http://www. smv.gob.pe/ConsultasP8/temp/Reporte_Sostenibilidad_Corporativa_2016.pdf 
